\title{
Quantum Monte Carlo simulation in the canonical ensemble at finite temperature
}

\author{
K. Van Houcke, ${ }^{1}$ S.M.A. Rombouts, ${ }^{1}$ and L. Pollet ${ }^{1}$ \\ ${ }^{1}$ Universiteit Gent - UGent, Vakgroep Subatomaire en Stralingsfysica \\ Proeftuinstraat 86, B-9000 Gent, Belgium
}

(Dated: September 10, 2018)

\begin{abstract}
A quantum Monte Carlo method with a non-local update scheme is presented. The method is based on a pathintegral decomposition and a worm operator which is local in imaginary time. It generates states with a fixed number of particles and respects other exact symmetries. Observables like the equal-time Green's function can be evaluated in an efficient way. To demonstrate the versatility of the method, results for the one-dimensional Bose-Hubbard model and a nuclear pairing model are presented. Within the context of the Bose-Hubbard model the efficiency of the algorithm is discussed.
\end{abstract}

PACS numbers: $02.70 . \mathrm{Ss}, 05.10 . \mathrm{Ln}, 21.60 . \mathrm{Ka}, 71.10 . \mathrm{Li}$

\section{INTRODUCTION}

Quantum Monte Carlo (QMC) simulation is a powerful and versatile method for the investigation of thermodynamic properties of many-body systems. When generating a Markovchain of configurations using a Metropolis scheme [1], it is known that updates based on local changes are inefficient, particularly near critical points. At transitional points this type of algorithm gives very large autocorrelation times, a phenomenon one refers to as 'critical slowing down' [2]. By developing non-local update schemes, this problem has been overcome for second order phase transitions. The Wolff single cluster algorithm [3] and the Swendsen-Wang multiple cluster algorithm [4], both used to solve classical physics problems, were successful applications of this idea. In the same spirit, loop algorithms [5, 6, 7] were developed for the study of discrete quantum systems. New configurations are obtained by flipping clusters in the form of loops. The systematic error caused by the the Suzuki-Trotter approximation [8, 9] was eliminated by formulating the world-line algorithms directly in continuous imaginary time [10, 11]. In the worm algorithm [12], the partition function is embedded in an extended configuration space, allowing a direct and exact evaluation of the one-body Green's function. The concept of non-local loop updates has also been implemented in the Stochastic Series Expansion (SSE) representation [13], leading to 'operator loop' update algorithms [14, 15] and 'directed loop' algorithms [16, 17], which are a further optimization of the loop construction. The general idea behind this is to construct moves in a locally optimal way [18].

All the non-local update world-line algorithms which are mentioned above sample the grand-canonical ensemble. In this way one can generate configurations with e.g. varying magnetization or occupation number. Results for the canonical ensemble are obtained by using only the configurations with the right particle number [19] or by rejecting loop updates which change this number [6]. It is clear that this is an inefficient way of working. Sampling the canonical ensemble directly would be advantageous when studying systems where particle-conservation is important. One example is the transition between the superfluid and the Mott phase in the BoseHubbard model at constant filling. This transition belongs to the $(\mathrm{d}+1)$-dimensional XY universality class [20]. When entering the superfluid phase, it becomes difficult to keep the number of bosons constant and tuning the chemical potential can become a very time-consuming task. When simulating mesoscopic systems like superconducting grains [21, 22] or atomic nuclei [23, 24], it is primordial to keep the number of particles constant. A world-line algorithm satisfying these conditions is presented [25] and discussed in detail in this paper. Besides particle-number conservation, the algorithm allows to include other symmetry-projections. It is constructed in such a way that all moves are accepted, which makes it efficient to run and easier to code. Though working in the canonical ensemble, our algorithm is still able to generate configurations with different winding numbers, in contrast to the local world-line method by Hirsch et al. [26]. We will demonstrate the versatility of the method by applying it to bosons and to paired fermions.

\section{THE ALGORITHM}

Practically all QMC methods are based on a decomposition of the evolution operator $e^{-\beta H}$. The trick is to break up this operator into pieces which can be handled exactly [27]. Generally one can write the Hamiltonian as consisting of an easy part $H_{0}$ and a residual interaction $V$,

$$
H=H_{0}-V
$$

The minus sign has been included to ease notations further on. For such a Hamiltonian, one can make an exact perturbative expansion in $V$ of the evolution operator, using the following integral expression [11]:

$$
\begin{aligned}
e^{-\beta H}= & \sum_{m=0}^{\infty} \int_{0}^{\beta} d t_{m} \int_{0}^{t_{m}} d t_{m-1} \cdots \int_{0}^{t_{2}} d t_{1} V\left(t_{1}\right) \\
& V\left(t_{2}\right) \cdots V\left(t_{m}\right) e^{-\beta H_{0}}
\end{aligned}
$$

with $V(t)=\exp \left(-t H_{0}\right) V \exp \left(t H_{0}\right)$ and $\beta$ the inverse temperature (also called imaginary time). The basic idea of the continuous-time loop algorithm [6, 10] and the worm algorithm [12] is to insert two adjoint world-line discontinuities. By propagating one of these discontinuities (which are called 
the mobile 'worm head' and stationary 'worm tail') through lattice space and imaginary time, the configuration changes in such a way that detailed balance is fulfilled. At that point one is not sampling according to the partition function $\operatorname{Tr}\left(e^{-\beta H}\right)$, but according to an extension hereof,

$$
\operatorname{Tr}\left(W^{\dagger} e^{-\tau H} W e^{-(\beta-\tau) H}\right),
$$

with $\tau$ the imaginary time interval between the worm 'head' operator $W^{\dagger}$ and 'tail' operator $W$. The worm head can be creating or annihilating, depending on the choice of $W$. After some Markov steps, the worm head bites its tail and the discontinuities are removed. Only configurations with continuous world-lines can contribute to the statistics according to $\operatorname{Tr}\left(e^{-\beta H}\right)$. In contrast to these algorithms, we will work with a worm which is local in imaginary time. The evolution operator extended by such a local worm (an imaginary timeindependent operator $A$ to be defined below) reads

$$
U^{\prime}(\beta, \tau)=e^{-\tau H} A e^{-(\beta-\tau) H},
$$

where $\tau$ can be regarded as the imaginary time at which the worm operator is inserted. We will show that by working with a local worm operator one can construct a very efficient sampling method, provided that the worm operator commutes with the residual interaction $(A V=V A)$. If $A$ furthermore commutes with the generators of a symmetry of $H_{0}$ and $V$, one can restrict the sampling to configurations with those specific symmetries, leading to symmetry-projected results. In particular one can sample the canonical ensemble with a worm operator that conserves particle number. We would like to emphasize at this point that the algorithm stated below does not depend on the specific structure of $A$. The operator $A$ has to be chosen in such a way that an ergodic Markov chain can be constructed, and therefore it will depend on the specific form of the interaction $V$.

If one decomposes the trace (restricted to the wanted particle number and symmetry) of $U^{\prime}(\beta, \tau)$ using Eq. (2) and inserts complete sets of eigenstates of $H_{0}$ at all imaginary times, one obtains a set of integrals which can be evaluated using Monte Carlo sampling. The Markov process will sample the configurations proportional to the weights

$$
\begin{aligned}
& W(m, i, t, \tau)=\left\langle i_{0}|V| i_{1}\right\rangle e^{-\left(t_{2}-t_{1}\right) E_{i_{1}}}\left\langle i_{1}|V| i_{2}\right\rangle e^{-\left(t_{3}-t_{2}\right) E_{i_{2}} \ldots} \\
& \left\langle i_{L-1}|V| i_{L}\right\rangle e^{-\left(\tau-t_{L}\right) E_{i_{L}}}\left\langle i_{L}|A| i_{R}\right\rangle e^{-\left(t_{R}-\tau\right) E_{i_{R}}\left\langle i_{R}|V| i_{R+1}\right\rangle \ldots} \\
& \left\langle i_{m-1}|V| i_{m}\right\rangle e^{-\left(t_{m}-t_{m-1}\right) E_{i_{m}}}\left\langle i_{m}|V| i_{0}\right\rangle e^{-\left(\beta+t_{1}-t_{m}\right) E_{i_{0}}}
\end{aligned}
$$

Each configuration is specified by an order $m$ (the number of interactions), a set $i$ of eigenstates of $H_{0}$ (with $i$ a shorthand notation for all the intermediate states $\left.\left|i_{0}\right\rangle, \ldots,\left|i_{L}\right\rangle,\left|i_{R}\right\rangle, \ldots,\left|i_{m}\right\rangle\right)$, interaction times $t_{1}, \ldots, t_{m}$, and the worm insertion time $\tau$. We use the notation $E_{i_{j}}=\left\langle i_{j}\left|H_{0}\right| i_{j}\right\rangle$. The configuration $\left|i_{L}\right\rangle$ to the left of the worm is changed by the worm operator into the configuration $\left|i_{R}\right\rangle$. We use the subscript $L(R=L+1)$ to indicate the eigenstate $\left|i_{L}\right\rangle\left(\left|i_{R}\right\rangle\right)$ and interaction time $t_{L}\left(t_{R}\right)$ just before (after) the worm operator in imaginary time. We will call the configurations for which $i_{L}=i_{R}$ diagonal configurations. By choosing the worm operator such that its diagonal elements are constant (i.e. $\langle i|A| i\rangle=c$ for all basis states $|i\rangle)$, the sum of the weights of all diagonal configurations is proportional to the particle-number projected trace of the evolution operator $U(\beta)$. This is nothing else than the canonical partition function $\operatorname{Tr}_{N}\left(e^{-\beta H}\right)$, with $\operatorname{Tr}_{N}$ the particle-number projected trace. Hence, sampling the configurations proportional to their weights $W(m, i, t, \tau)$ leads to a sampling of the canonical ensemble. The Markov process is set up using the Metropolis-Hastings algorithm [1, 28], hereby sampling in an extended space according to $\operatorname{Tr}_{N}\left[U^{\prime}(\beta, \tau)\right]$. At each Markov step only a few of the factors of Eq. (5) are altered by the worm operator which moves to a new point in imaginary time. These worm operator moves will be constructed in a such a way that detailed balance is fulfilled locally at each Markov step. Therefore it is also fulfilled when going from one diagonal configuration to another. It takes a number of Markov steps before diagonal observables (i.e. observables which commute with $H_{0}$ ) can be measured again. While each Markov step contains only local changes, the chain of steps between two diagonal configurations corresponds to a global update. Non-diagonal operators can be measured using the fact that one samples in an extended space. By keeping track of the worm moves between two diagonal configurations, statistics for the expectation values of nondiagonal operators can be collected, similar to the way one evaluates the one-body Green's function in the worm algorithm [12]. Our method however will lead to much better statistics for equal-time non-diagonal operators, because the worm operator is always local in imaginary time.

Before shifting the worm operator over some imaginary time interval $\Delta \tau$, a direction $D$ has to be chosen. One can choose between the directions $D=R$ (higher values of $\tau$ ) and $D=L$ (lower values of $\tau$ ) with some probability $P(D)$, to be specified later. The presence of the exponentials in Eq. (5) inspires us to choose the time shift $\Delta \tau$ proportional to an exponential distribution,

$$
P(\Delta \tau) d \Delta \tau=\varepsilon_{D} e^{-\varepsilon_{D} \Delta \tau} d \Delta \tau,
$$

with $\varepsilon_{D}$ an optimization parameter. In shifting the worm operator from $\tau$ to a new imaginary time $\tau^{\prime}=\tau+\Delta \tau$, the worm operator can encounter an interaction operator $V$ at some time in between. Assume the direction $R$ is chosen and the worm operator meets an interaction at time $t_{R}$. Let us first consider the situation where the worm operator moves through this interaction, without annihilating it,

$$
\left\langle i_{L}|A| i_{R}\right\rangle\left\langle i_{R}|V| i_{R+1}\right\rangle \longrightarrow\left\langle i_{L}|V| i_{R}^{\prime}\right\rangle\left\langle i_{R}^{\prime}|A| i_{R+1}\right\rangle .
$$

When passing the interaction, the intermediate state can change. A convenient way to pick one of these possible changes is to choose the new configuration proportional to its weight

$$
P_{R+1, L}\left(i_{R}^{\prime}\right)=\frac{\left\langle i_{L}|V| i_{R}^{\prime}\right\rangle\left\langle i_{R}^{\prime}|A| i_{R+1}\right\rangle}{\left\langle i_{L}|V A| i_{R+1}\right\rangle}
$$

Part (a) of Figure 1 shows a diagrammatic representation of the different ways in which a general one-body worm operator $A=\sum_{i, j} c_{i j} a_{i}^{\dagger} a_{j}$ (for some constants $c_{i j}$ ) can pass a similar 
interaction $V$. The worm operator is represented by a curly line and the interaction by a vertical straight line. For this type of worm and interaction operator there are always at most four ways in which the intermediate state can change. It should be noted that our choice Eq. [8] is not unique and possibly more optimal choices can be found [18]. Because of this choice however, there appears a factor

$$
\begin{aligned}
n_{R+1, L} & =\frac{\left\langle i_{L}|V| i_{R}^{\prime}\right\rangle\left\langle i_{R}^{\prime}|A| i_{R+1}\right\rangle P_{L, R+1}\left(i_{R}\right)}{\left\langle i_{L}|A| i_{R}\right\rangle\left\langle i_{R}|V| i_{R+1}\right\rangle P_{R+1, L}\left(i_{R}^{\prime}\right)} \\
& =\frac{\left\langle i_{L}|V A| i_{R+1}\right\rangle}{\left\langle i_{L}|A V| i_{R+1}\right\rangle}
\end{aligned}
$$

in the acceptance factor of the Metropolis-Hastings algorithm. Every time the worm operator passes an interaction an analogous factor appears, depending on the direction $D$ of propagation. Therefore it's advantageous to impose on $A$ the condition,

$$
A V-V A=0,
$$

because then $n_{D D^{\prime}}=1$ in all cases, and we do not have to worry about this normalization factor anymore. Furthermore, Eq. (10) ensures that the worm operator can always pass the interaction it encounters. If one would choose a worm operator $A$ that does not satisfy this condition, as in grand-canonical algorithms, it is possible the worm operator cannot pass the interaction and the direction of propagation has to change, in that way undoing changes previously made. It is intuitively clear that these 'bounces' give rise to a slow decorrelation and should be avoided [16, 17, 18]. In the directed loop algorithm one increases the efficiency of the loop update by minimizing the appearance of bounces, but they cannot be eliminated completely because of the reversibility condition. In what follows we will assume the condition Eq. (10) is fulfilled, making the algorithm bounce-free. We will drop the factors $n_{D D^{\prime}}$ to ease the equations. After passing through the interaction at time $t_{D}$, one has to choose a new imaginary-time shift $\Delta \tau$. However, one can avoid generating a new random number by taking the new shift as follows:

$$
\Delta \tau=\left(\tau^{\prime}-t_{D}\right) \frac{\left(\varepsilon_{D}\right)_{\text {old }}}{\left(\varepsilon_{D}\right)_{\text {new }}},
$$

where the parameter $\varepsilon_{D}$ has been updated after passing the interaction.

The choice of the parameters $\varepsilon_{D}$ follows from detailed balance. Because the time shifts $\Delta \tau$ of the worm operator are chosen by Eqs. (6) and (11), adding the constraint

$$
E_{R}-E_{L}=\varepsilon_{L}-\varepsilon_{R},
$$

ensures that all the exponentials which appear in the acceptance factor of the Metropolis-Hastings algorithm cancel, leading to an efficient sampling method. So in practice one can choose any positive value for $\varepsilon_{L}$ and $\varepsilon_{R}$, as long as Eq. 12] is fulfilled at each step of the worm movement. To conclude, we write down the acceptance factor for the above procedure when the worm operator does not change the number of interactions,

$$
\begin{aligned}
q & =\frac{W\left(m, i^{\prime}, t^{\prime}, \tau^{\prime}\right) P\left(i^{\prime}, t^{\prime}, \tau^{\prime} \rightarrow i, t, \tau\right)}{W(m, i, t, \tau) P\left(i, t, \tau \rightarrow i^{\prime}, t^{\prime}, \tau^{\prime}\right)} \\
& =\frac{\left(\varepsilon_{D^{\prime}}\right)_{\text {initial }}}{\left(\varepsilon_{D}\right)_{\text {final }}},
\end{aligned}
$$

where $\left(\varepsilon_{D}\right)_{\text {final }}\left(\left(\varepsilon_{D^{\prime}}\right)_{\text {initial }}\right)$ is the value of $\varepsilon_{D}\left(\varepsilon_{D^{\prime}}\right)$ at the end (beginning) of the worm operator move into direction $D$, and $D^{\prime}$ denotes the opposite direction of $D$. The actual acceptance probability is given by $\min (1, \mathrm{q})$, according to the MetropolisHastings algorithm.

Let us now introduce a number of steps, which allow to change the number of interactions in a reversible way. We want the acceptance factor of such updates to be local, i.e. the probability to pass, create or annihilate an interaction should only depend on the properties of the state at that point in imaginary space-time. We consider three extensions of the procedure outlined above where no interactions are created or deleted:

- At the beginning of the Markov step, we introduce the possibility that the worm operator creates a new interaction with probability $c_{D}$, which depends on the direction $D$ of propagation. This creation will also change the intermediate state:

$$
\left\langle i_{L}|A| i_{R}\right\rangle \longrightarrow\left\langle i_{L}|V| i^{\prime}\right\rangle\left\langle i^{\prime}|A| i_{R}\right\rangle,
$$

assuming again the worm operator is moving in the $D=R$ direction. The new intermediate state $\left|i^{\prime}\right\rangle$ will be chosen with probabilities

$$
P_{R L}\left(i^{\prime}\right)=\frac{\left\langle i_{L}|V| i^{\prime}\right\rangle\left\langle i^{\prime}|A| i_{R}\right\rangle}{\left\langle i_{L}|V A| i_{R}\right\rangle} .
$$

For a worm operator move in the $D=L$ direction, probabilities $P_{L R}\left(i^{\prime}\right)$ can be defined in an analogous way. Figure 11 (parts (b) and (c)) shows a diagrammatic representation of the insertion of a one-body interaction at the beginning of the worm move. For a diagonal configuration only the diagonal part of $A$ contributes to the matrix element $\left\langle i_{L}|A| i_{R}\right\rangle$. In this situation the worm operator is represented by little circles and all world-lines are continuous. We will call this the 'diagonal worm'.

- When the worm operator arrives at an interaction, one also has to consider the possibility of annihilating that interaction. Suppose the interaction can be deleted. Let $a_{D}$ be the probability to annihilate the interaction while continuing the worm update, and $s_{D}$ the probability to annihilate the interaction and stop the worm update. Then $1-a_{D}-s_{D}$ is the probability to pass through that interaction and continue the worm operator.

- To maintain reversibility, one also has to include the possibility that the construction of the Markov step does not halt at the moment the worm operator has finished a shift $\Delta \tau$ without encountering an interaction. At that point one has to choose between stopping the worm operator, or to continue, with the possibility of inserting a 
new interaction at that point. Let $f_{D}$ be the probability to continue the worm operator without inserting an interaction, $g_{D}$ the probability to insert an interaction and to continue the worm operator, then $1-f_{D}-g_{D}$ will be the probability to stop the worm operator, without inserting an interaction.

After creating, annihilating or passing an interaction, a new time shift $\Delta \tau$ should again be chosen according to Eqs. (6) or (11). Note that the parameter $f_{D}$ is redundant: jumping with a parameter $\varepsilon_{D}$ and continuing the worm operator unaltered with probability $f_{D}$ is statistically equivalent to making a jump with parameter $\varepsilon_{D}\left(1-f_{D}\right)$, and then choosing between either stopping the worm operator or inserting an interaction and move on. Therefore, one can set $f_{D}=0$ without loss of generality. We now determine how the other parameters should be chosen in order to satisfy detailed balance. Assume a direction $D$ is chosen. When no interaction is inserted at the beginning of the worm move, a factor

$$
q_{D}^{0}=\frac{\varepsilon_{D^{\prime}}\left(1-g_{D^{\prime}}\right)}{1-c_{D}},
$$

appears in the acceptance factor. If on the other hand an interaction is created at the initial time $\tau$ of the worm operator, this will lead to a factor

$$
q_{D}^{c}=\frac{\mathcal{N}_{D D^{\prime}} s_{D^{\prime}}}{c_{D}}
$$

with

$$
\mathcal{N}_{D D^{\prime}}=\frac{\left\langle i_{D}|V A| i_{D^{\prime}}\right\rangle}{\left\langle i_{D}|A| i_{D^{\prime}}\right\rangle} .
$$

A new intermediate state is chosen with probabilities Eq. (15). At the end of the Markov step, the worm operator will annihilate an interaction or not, leading to extra factors in the global acceptance factor which have the inverse form of Eqs. (17) and (16), because of the inverse symmetry between beginning and end of the move. At intermediate points, we can encounter the following situations. The worm operator can stop after a shift $\Delta \tau$ between two interactions, insert an interaction and move on. The inverse situation of this can also occur, when an interaction is annihilated and the worm operator moves on. Or the worm operator can simply pass an interaction without annihilating it. In order to have a good total acceptance factor, we will require that these intermediate steps do not contribute to the acceptance factor. This condition leads to the constraints

$$
\begin{aligned}
\mathcal{N}_{D D^{\prime}} a_{D^{\prime}} & =\varepsilon_{D} g_{D}, \\
a_{D}+s_{D} & =a_{D^{\prime}}+s_{D^{\prime}} .
\end{aligned}
$$

Apart from that, we want the sampling to be as uniform as possible, which suggests the condition $q_{D}^{0}=q_{D}^{c}$. Putting all this together, the total acceptance factor is given by

$$
\begin{aligned}
q & =\frac{W\left(m^{\prime}, i^{\prime}, t^{\prime}, \tau^{\prime}\right) P\left(m^{\prime}, i^{\prime}, t^{\prime}, \tau^{\prime} \rightarrow m, i, t, \tau\right)}{W(m, i, t, \tau) P\left(m, i, t, \tau \rightarrow m^{\prime}, i^{\prime}, t^{\prime}, \tau^{\prime}\right)} \\
& =\frac{\left(q_{D}\right)_{\text {initial }}}{\left(q_{D^{\prime}}\right)_{\text {final }}}
\end{aligned}
$$

where

$$
q_{D}=\varepsilon_{D^{\prime}}+\mathcal{N}_{D D^{\prime}}\left(s_{D^{\prime}}-a_{D}\right) .
$$

The factor $\left(q_{D}\right)_{\text {initial } / \text { final }}$ has to be evaluated at the beginning and the end of the Markov step in direction $D$ (with $D^{\prime}$ the opposite of $D$ ). The creation probability is given by Eq. (17),

$$
c_{D}=\frac{\mathcal{N}_{D D^{\prime}} s_{D^{\prime}}}{q_{D}} .
$$

We still have to determine how to choose the direction $D$. The acceptance ratio of Eq. (21) inspires us to choose the direction of the move with probabilities

$$
\begin{aligned}
& P(D=R)=\frac{q_{R}}{R_{L R}}, \\
& P(D=L)=\frac{q_{L}}{R_{L R}} .
\end{aligned}
$$

with $R_{L R}=q_{R}+q_{L}$. By accepting all worm operator moves a distribution given by

$$
W^{\prime}(m, i, t, \tau)=R_{L R} W(m, i, t, \tau),
$$

will be sampled, instead of the distribution $W(m, i, t, \tau)$. Because the factors $R_{L R}$ fluctuate only mildly in practice, accepting all moves still leads to a a very useful sampling method. It speeds up the algorithm and reduces the complexity of the code. Finite temperature observables can still be evaluated by taking the extra weighting factor into account:

$$
\begin{aligned}
\langle Q\rangle_{\beta} & =\frac{\operatorname{Tr}\left[e^{-\beta\left(H_{0}-V\right)} Q\right]}{\operatorname{Tr}\left[e^{-\beta\left(H_{0}-V\right)}\right]} \\
& =\frac{\sum_{s \in \mathcal{S}}\left(Q_{s} /\left(R_{L R}\right)_{s}\right)}{\sum_{s \in \mathcal{S}}\left(1 /\left(R_{L R}\right)_{s}\right)} .
\end{aligned}
$$

Each time the worm operator creates, annihilates or passes an interaction, the parameters $\varepsilon_{D}, c_{D}, a_{D}, s_{D}, g_{D}$ are determined by Eqs. (12), 20, 22) and (23). This still leaves a lot of freedom. We will focus here on two limiting cases. First we will consider the case where one of the two parameters $\varepsilon_{R}$ and $\varepsilon_{L}$ is always zero. In that way it can occur the time shift $\Delta \tau$ becomes infinite. This amounts to the worm operator directly jumping to the next interaction, which speeds up decorrelation in imaginary time direction. In order to obtain a worm move that changes the configurations as much as possible, the parameters $g_{L}, g_{R}, a_{L}$ and $a_{R}$ are maximized. The set of parameters obtained in this way is shown in Table $\prod$ for the case $E_{R}>E_{L}$. We will call this solution A. The solution for the case $E_{L}>E_{R}$ can be found by interchanging the subscripts $L$ and $R$. Note that in this solution the worm operator always starts to move into the direction of the highest diagonal energy. It is clear that whenever the worm operator is moving in the direction of the highest diagonal energy or whenever $E_{R}=E_{L}$, the time shift $\Delta \tau$ becomes infinite. There are a number of extra conditions one should keep in mind. Assume the worm operator starts to move in the direction $D=R$ (because $\left.E_{R}>E_{L}\right)$. When the worm operator arrives at an interaction that can be annihilated, one has to determine $E_{L}, E_{R}$ and $\mathcal{N}_{L R}$ 


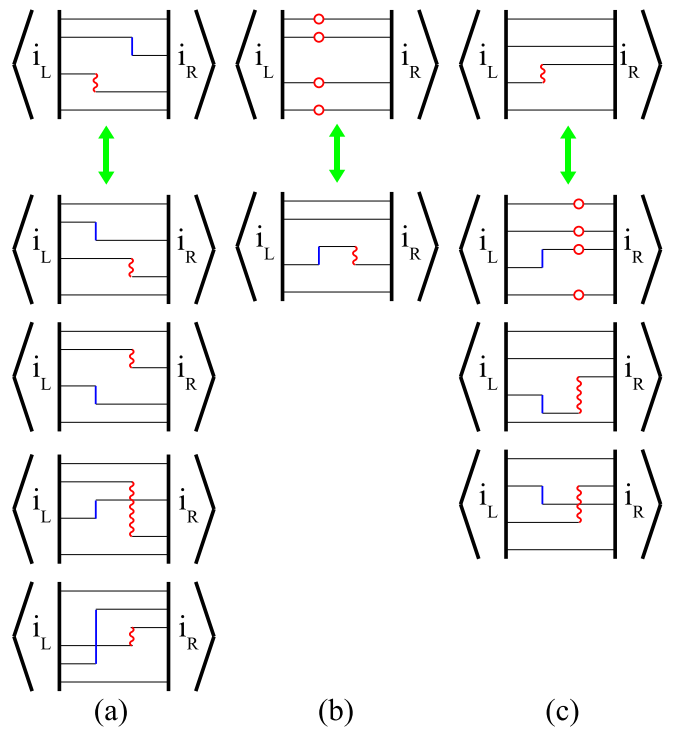

FIG. 1: A diagrammatic representation for one-body worm operator moves. The worm operator is represented by a curly line and the interaction $V$ by a solid vertical line. We distinguish between the following updates. (a) When the worm operator moves in the $D=R$ direction, it can encounter some interaction. The worm operator can pass the interaction, in that way changing the intermediate state. For general one-body worm and interaction operators, there are at most four possible ways in doing this. (b)-(c) At the beginning of the worm move, we introduce the possibility of inserting an interaction. When the initial worm is diagonal (represented by circles), a number of interaction insertions of the type shown in (b) are possible. In part (c) the initial worm operator is not diagonal, and an interaction insertion can make the worm diagonal or not.

after the annihilation. If $E_{R}>E_{L}$ is still satisfied then, $s_{R}$ and $a_{R}$ from Table \are the correct probabilities to stop or continue the worm operator. If now $E_{L}>E_{R}$ on the other hand, one has to use the solution $s_{R}=\min \left(1, \frac{\mathrm{E}_{\mathrm{L}}-\mathrm{E}_{\mathrm{R}}}{\mathcal{N}_{\mathrm{CR}_{R}}}\right)$ and $a_{R}=0$, but the worm operator keeps moving in the same direction. The time shift of the worm operator is only finite when it moves in the direction $D$ and $E_{D}<E_{D}^{\prime}$. Note also that only $g_{L}$ is mentioned in Table $\llbracket$ because the parameter $g_{D}$ has only meaning when the time shift is finite. In the present solution however, a problem arises whenever $E_{L}=E_{R}$. In this case $\varepsilon_{R}=\varepsilon_{L}=0$ and the time shift $\Delta \tau$ is always infinite. Because $s_{R}=s_{L}=0$ in addition, the worm operator never halts. As a consequence configurations with a diagonal worm will never be sampled. This can be solved by proposing a small but finite stopping probability. This alternative solution for the case $E_{L}=E_{R}$ is also given in Table【 The global parameter $\phi$ should be taken small (such that $0<\phi<\mathcal{N}_{D D^{\prime}}$ for all diagonal configurations) but not zero, and can be chosen in order to optimize the decorrelation between successive evaluations of observables. Note $R_{L R}$ of Eq. 26) takes the constant value $2 \phi$.

Another possibility to find algorithm parameters follows from the idea that we want the step size $\Delta \tau$ to be of the order of the mean imaginary time interval between two interactions.

\begin{tabular}{|c||c|c|}
\hline \hline parameters & $\begin{array}{c}\text { diagonal configurations } \\
\left(i_{L}=i_{R}\right) \\
\left(E_{L}=E_{R}\right)\end{array}$ & $\begin{array}{c}\text { diagonal configurations } \\
\left(i_{L} \neq i_{R}\right) \\
\left(E_{L}<E_{R}\right)\end{array}$ \\
\hline \hline$\varepsilon_{R}$ & 0 & 0 \\
$\varepsilon_{L}$ & 0 & $E_{R}-E_{L}$ \\
$q_{R}$ & $\phi$ & $E_{R}-E_{L}$ \\
$q_{L}$ & $\phi$ & 0 \\
$c_{R}$ & 1 & $\min \left(1, \frac{\mathcal{N}_{L R}}{E_{R}-E_{L}}\right)$ \\
$c_{L}$ & 1 & 0 \\
$s_{R}$ & $\frac{\phi}{\mathcal{N}_{L}}$ & 0 \\
$s_{L}$ & $\frac{\phi}{\mathcal{N}_{L R}}$ & $\min \left(1, \frac{E_{R}-E_{L}}{\mathcal{N} \mathcal{N}_{R}}\right)$ \\
$a_{R}$ & 0 & $\min \left(1, \frac{E_{R}-E_{L}}{\mathcal{V}_{L R}}\right)$ \\
$a_{L}$ & 0 & 0 \\
$g_{L}$ & 0 & $\min \left(1, \frac{\mathcal{V}_{L R}}{E_{R}-E_{L}}\right)$ \\
$R_{L R}$ & $2 \phi$ & $E_{R}-E_{L}$ \\
\hline \hline
\end{tabular}

TABLE I: A set of algorithm parameters satisfying Eqs. [12, 20, 22] and 23] for the cases $E_{L}=E_{R}$ and $E_{L}<E_{R}$ (otherwise interchange the subscripts $L$ and $R$ ). We call this solution A, for which one of the parameters $\varepsilon_{D}$ is always zero.

\begin{tabular}{|c||c|c|}
\hline \hline parameters & $\begin{array}{c}\text { diagonal configurations } \\
\left(i_{L}=i_{R}\right) \\
\left(E_{L}=E_{R}\right)\end{array}$ & $\begin{array}{c}\text { diagonal configurations } \\
\left(i_{L} \neq i_{R}\right) \\
\left(E_{L}<E_{R}\right)\end{array}$ \\
\hline \hline$\varepsilon_{R}$ & $\mathcal{N}_{L R}$ & $\mathcal{N}_{L R}$ \\
$\varepsilon_{L}$ & $\mathcal{N}_{L R}$ & $\mathcal{N}_{L R}+E_{R}-E_{L}$ \\
$q_{R}$ & $\mathcal{N}_{L R}-E_{L}$ \\
$q_{L}$ & $\mathcal{N} L R$ & 0 \\
$c_{R}$ & $\phi$ & 0 \\
$c_{L}$ & $\phi$ & 0 \\
$s_{R}$ & $\phi$ & 0 \\
$s_{L}$ & $\phi$ & 0 \\
$a_{R}$ & $\phi$ & 1 \\
$a_{L}$ & $\phi$ & 1 \\
$g_{R}$ & $\phi$ & 1 \\
$g_{L}$ & $\phi$ & $\mathcal{N}_{L R}$ \\
$R_{L R}$ & $2 \mathcal{N}_{L R}$ & $E_{R}-E_{L}$ \\
\hline \hline
\end{tabular}

TABLE II: An alternative set of parameters for which one of the parameters $\varepsilon_{D}$ is always $\mathcal{N}_{L R}$. We call this solution $\mathrm{B}$. The parameter $\phi$ can be chosen to optimize the algorithm.

Therefore we consider the case where one of the two parameters $\varepsilon_{R}$ and $\varepsilon_{L}$ is $\mathcal{N}_{L R}$. As a consequence the time shift is always finite. For $E_{R}>E_{L}$ such a set of parameters is given in Table I Again, the case $E_{L}=E_{R}$ needs an alternative solution, since otherwise $R_{L R}$ would be zero for diagonal configurations. We will refer to this solution as solution $\mathrm{B}$.

In short, the algorithm is based on a time-dependent perturbation in the interaction $V$ (see Eq. (2)), so there is no systematic error arising from time discretization. Because we choose time shifts of a worm operator according to Eq. (6) the diagonal part of the Hamiltonian is handled exactly. There are a number of algorithm parameters, which have to satisfy Eqs. (12), (20), (22) and (23). We have derived two sets of algorithm parameters, satisfying these equations. In the first set (solution $\mathrm{A}$ ) one of the parameters $\varepsilon_{D}$ is always zero and 
in the second (solution B) it is equal to $\mathcal{N}_{L R}$. Therefore the main difference between these two solutions will be the size of the imaginary time shift $\Delta \tau$. Other algorithms where $\varepsilon_{R}$ or $\varepsilon_{L}$ take values between 0 and $\mathcal{N}_{L R}$ can be constructed in a similar way, taking for now only these limiting cases. In the next section our QMC algorithm will be applied to the BoseHubbard model. The efficiency of the two different solutions leading to different algorithms will be compared in this context.

\section{APPLICATION TO THE BOSE-HUBBARD MODEL}

Ultracold bosonic atoms in an optical lattice are described by the Bose-Hubbard model [29, 30, 31],

$$
H=-t \sum_{\langle i, j\rangle}^{N_{s}} b_{i}^{\dagger} b_{j}+\frac{U}{2} \sum_{i}^{N_{s}} n_{i}\left(n_{i}-1\right)
$$

with $b_{i}^{\dagger}\left(b_{i}\right)$ the boson creation (annihilation) operator on site $i, n_{i}$ the number operator on site $i$ and $\langle i, j\rangle$ denoting nearest neighbors. The lattice has $N_{s}$ sites, occupied by $N$ bosons. The parameter $t$ is the tunneling amplitude and $U$ is the onsite repulsion strength. We will restrict the discussion here to the one-dimensional homogeneous case without trap. At low values of $U / t$ the system forms a compressible superfluid. This phase is characterized by a gapless excitation spectrum and long-range phase coherence. By increasing $U / t$, a quantum phase transition from a superfluid state to a Mott insulating state is achieved at integer densities. In the pure Mott phase the bosons are localized at individual lattice sites and all phase coherence is lost due to quantum fluctuations. In addition, density fluctuations disappear when entering the Mott phase and a gap appears in the excitation spectrum. This phase driven transition belongs to the Berezinskii-KosterlitzThouless (BKT) [32, 33] universality class in one dimension. The Bose-Hubbard Hamiltonian can be rewritten in the form Eq. (1),

$$
\begin{aligned}
H_{0} & =\frac{U}{2} \sum_{i} n_{i}\left(n_{i}-1\right), \\
V & =t \sum_{\langle i, j\rangle} b_{i}^{\dagger} b_{j} .
\end{aligned}
$$

As argued above, it is advantageous to take the worm operator $A$ such that it commutes with $V$. An operator that satisfies this condition is given by

$$
A=\frac{1}{\bar{N}} \sum_{i} n_{i}+\sum_{i \neq j} b_{i}^{\dagger} b_{j}
$$

with $\bar{N}$ a c-number to be optimized. In our calculations this parameter is always set equal to the total number of particles. We have checked our code by comparing with exact diagonalization results for small lattices. Ergodicity was tested numerically. Power law behavior of correlation functions coincides with predictions from bosonization theory for large lattices.
The one-body Green's function $G(r)=\left\langle b_{i}^{\dagger} b_{i+r}\right\rangle$ is calculated by updating the entry $r$ in a histogram for $G(r)$ at every Markov step. The function $G(r)$ can be normalized directly from the diagonal / non-diagonal worm fraction. The non-diagonal worm components $b_{i}^{\dagger} b_{i+r}$ of Eq. (30) can be given a different weight, leading to a worm matrix representation of the symmetric Toeplitz type (i.e. a symmetric matrix with constant values along negative-sloping diagonals). Such a worm operator still commutes with the interaction part $V$ of the Hamiltonian. By giving some worm components a bigger weight, the corresponding components $G(r)$ will be updated more often, leading to a higher accuracy and mimicking flat histograms [34]. The condensed fraction $\rho_{c}$ can be determined via

$$
\rho_{c}=\frac{1}{N N_{s}} \sum_{i, j}^{N_{s}}\left\langle b_{i}^{\dagger} b_{j}\right\rangle .
$$

The superfluid fraction can be determined using the winding number [35],

$$
\rho_{s}=\frac{\left\langle W^{2}\right\rangle N_{s}^{2}}{2 t N \beta}
$$

where $\left\langle W^{2}\right\rangle$ is the mean square of the winding number operator in one dimension. Figure 2 shows the condensed and superfluid fraction for a uniform one-dimensional system of 128 sites at a density of exactly one particle per site. Calculations were performed at an inverse temperature $\beta=128 t^{-1}$, using the algorithm based on solution $\mathrm{A}$. We have used the algorithm to study the quantum critical behavior of the one-dimensional Bose-Hubbard model with constant filling, using Renormalization Group flow equations. Studying the BKT transition is notoriously difficult because of the logarithmic finite-size corrections. The present algorithm has the big advantage of keeping the density constant, in contrast to the grand-canonical approaches. The results of this study will be presented elsewhere [36].

We compare the efficiencies of solutions A and B. To this purpose, we simulate a one-dimensional lattice with 32 sites at an inverse temperature $\beta=32 t^{-1}$ and a filling factor of one boson per site. The simulations consisted of 40 Markov chains that each ran 600 seconds after thermalization on a Pentium III processor. The same code was used, with only minor changes to go from algorithm A to B. We discuss the efficiency by looking at the standard deviations on the expectation value of $V$ of Eq. 29] and on the average squared density. We calculated the squared density $n^{2}$ by averaging over all sites,

$$
\left\langle n^{2}\right\rangle=\frac{1}{N_{s}} \sum_{i}^{N_{s}}\left\langle n_{i}^{2}\right\rangle .
$$

The expectation value of the interaction term $V$ was not calculated via the correlation function $G(1)$, but by counting the number of interaction vertices in the configuration whenever the worm operator was diagonal [37]. When looking at the standard deviation on the squared density (Figure 3, one can conclude solution $\mathrm{A}$ is the most efficient one. We found a similar picture when looking at the standard deviations on the expectation value of $H_{0}$ of Eq. 29. The errors on the standard 


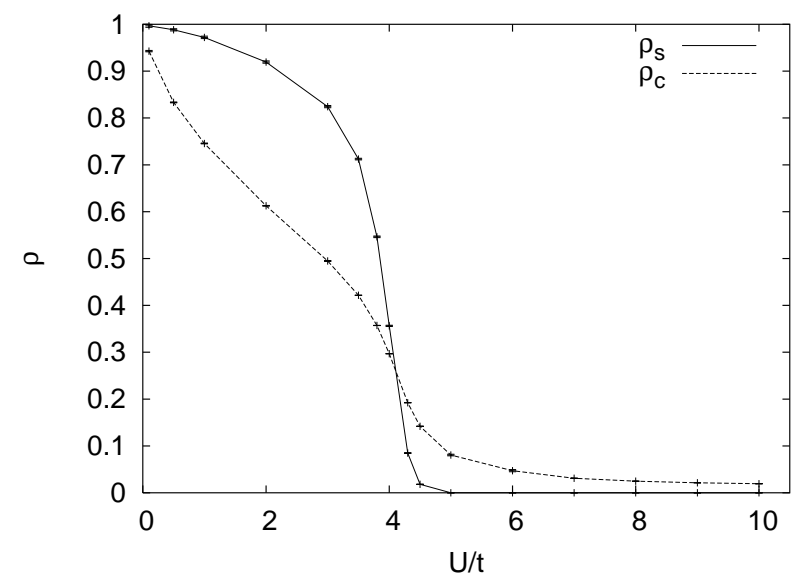

FIG. 2: Superfluid $\left(\rho_{s}\right)$ and condensed fraction $\left(\rho_{c}\right)$ for the onedimensional homogeneous Bose-Hubbard model as a function of $U / t$. The fractions have been calculated for a uniform lattice with $N_{s}=128$ at an inverse temperature $\beta=128 t^{-1}$, using Eqs. 31 and 32. The condensed fraction was calculated from the correlation function $\left\langle b_{i}^{\dagger} b_{i+r}\right\rangle$, for which we have high statistics.

deviations lie within ten percent. From the standard deviation on the expectation value of the interaction term $V$ (Figure 4), it follows that solution B is better in the superfluid phase. The same conclusion follows from the total energy. For the condensed fraction the deviations are smallest for solution A for all values of $U / t$. Those on the superfluid fraction lie very close for solution A and B (see Figure 5). We found that varying the parameter $\phi$ of Tables $\square$ and $\Pi$ does not change the efficiency in a significant way, as long as $\phi$ is not too small. For further simulations we will always choose the parameter $\phi$ as big as possible, under the constraint $\phi \leq \mathcal{N}_{D D^{\prime}}$. Figures 3 4 and 5 show also standard deviations resulting from the directed loop SSE algorithm [16, 17, 18]. One has to be very careful when comparing efficiencies of different algorithms. First, the SSE code works in the grand-canonical ensemble. In the SSE simulations, the chemical potential was changed in such a way that $N$ remained constant. Second, the efficiency does not only depend on the algorithm, but also on the used data structures. In a SSE approach, the decomposition of the evolution operator corresponds to a perturbation expansion in all terms of the Hamiltonian, while the decomposition Eq. (2) perturbs only in the off-diagonal terms $V$. For the Bose-Hubbard model, where the contribution of the diagonal terms is large, the last approach is preferable. For all calculated observables the standard deviations resulting from the SSE code were the largest. Figures 3 and 4 show the SSE deviations increase rather rapidly with increasing $U / t$, whereas the deviations resulting from our method remain of the same order. We also calculated autocorrelation times for different observables. Here each bin ended after a constant number of measurements. We noticed that for solution B the autocorrelation times became very big for high values of $U / t$. For small $U / t$, the autocorrelation time for solution $\mathrm{A}$ is of the order of the number of Markov steps needed for 10 diagonal updates, and increases only slowly with increasing $U / t$. Of course it should be noted the algorithm based on solution A had to run much longer in order to get the same number of diagonal measurements. For all measured observables we found similar autocorrelation times.

We conclude that solution A, derived in the previous section, is more efficient than solution B, except in the superfluid phase when looking at the interaction energy $V$. This can be understood by remarking that the time shifts of the worm operator are much larger for solution A. In the algorithm based on solution $\mathrm{B}$, the time shifts are of the order of the mean imaginary time between two interaction vertices in the configurations. This explains why the standard deviations on the interaction energy are smallest for this solution in the superfluid phase. We also conclude that our algorithm is more efficient than the directed loop SSE algorithm when simulating the one-dimensional Bose-Hubbard model. In the next section, we will apply the algorithm to a pairing model. In what follows, all calculations are performed using the algorithm based on solution B.

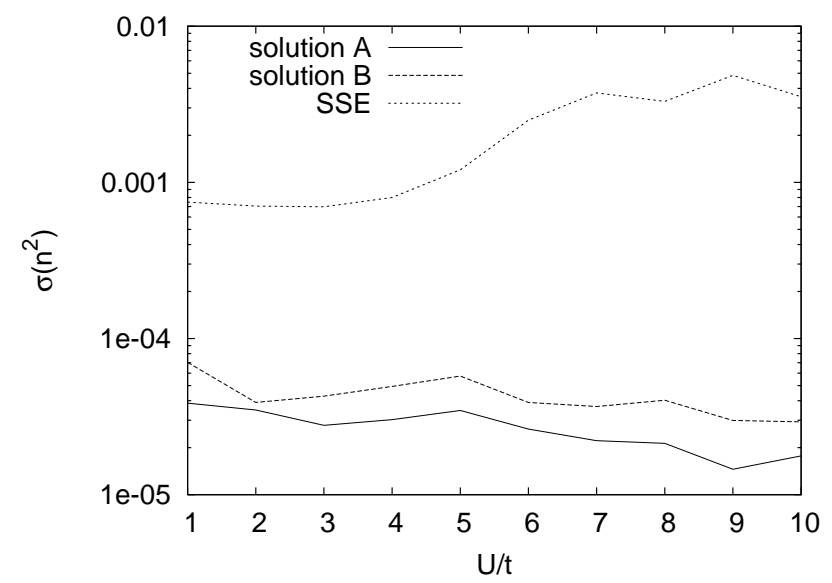

FIG. 3: A comparison between the standard deviations on the squared density (see Eq. 33) resulting from the directed loop SSE algorithm and the algorithms based on solutions A and B. The homogeneous Bose-Hubbard model is simulated for a lattice with 32 sites and 32 bosons at an inverse temperature $\beta=32 t^{-1}$. The deviations resulted after a QMC calculation with 40 independent Markov chains that each ran 600 seconds on a Pentium III processor.

\section{APPLICATION TO A PAIRING MODEL}

In the nuclear shell model, quantum Monte Carlo methods are valuable because they offer the possibility of doing calculations in much larger model spaces than conventional diagonalization techniques. Finite temperature shell model studies have been done with the aid of auxiliary-field QMC methods [23], and ground-state properties of light nuclei have been calculated using variational and diffusion QMC techniques [38]. Furthermore, being able to calculate thermal properties of nuclei makes it in principle possible to calculate nuclear level 


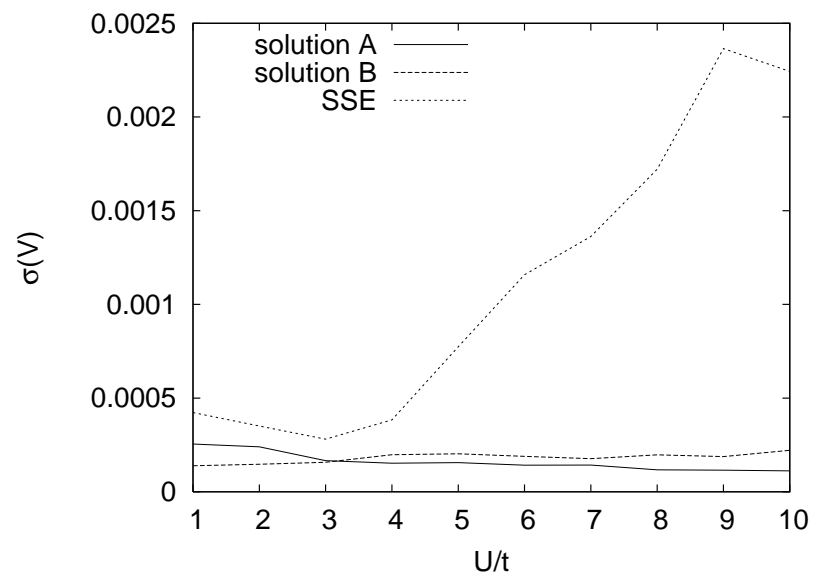

FIG. 4: The standard deviation on the mean value of $V$ (see Eq. 29 for the homogeneous Bose-Hubbard model as a function of $U / t$. Here solution A is the most efficient one in the Mott phase. In the superfluid phase, solution B becomes more efficient.

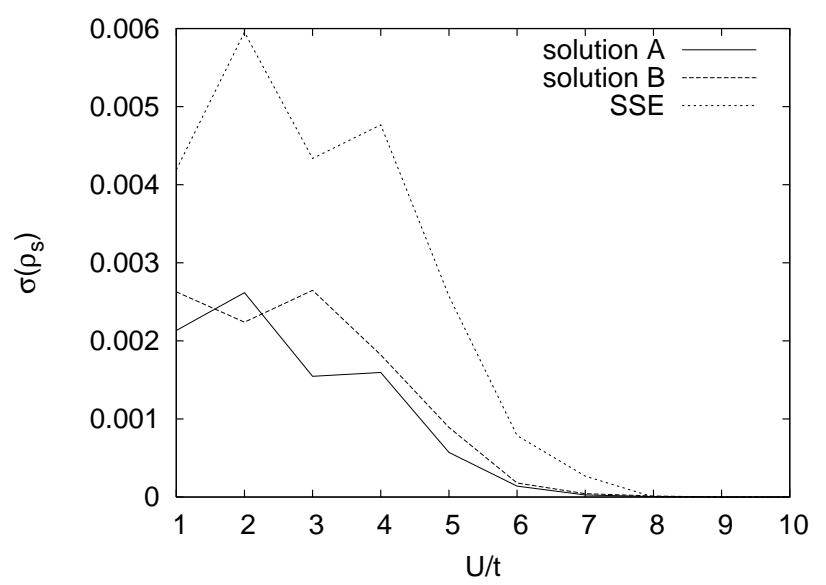

FIG. 5: The standard deviation on the mean value of the superfluid fraction $\rho_{s}$. The deviations result from solutions $\mathrm{A}$ and $\mathrm{B}$, and the directed loop SSE method. Each simulation consisted of 40 independent Markov chains that each ran 600 seconds.

densities [39]. These densities are extremely important for making good theoretical estimates of nuclear reaction rates.

The basic assumption in the shell model is the presence of a mean field in which the nucleons move. To improve on this, a residual interaction between the nucleons is introduced. Pairing between nucleons is the main short-range correlation induced by the residual interaction. Adding a simple pairing Hamiltonian to the mean-field Hamiltonian

$$
H_{m f}+H_{P}=\sum_{t=p, n} \sum_{k} e_{k t} n_{k t}-\sum_{t=p, n} \frac{G_{t}}{4} \sum_{k, k^{\prime}} a_{k^{\prime} t}^{\dagger} a_{\overline{k^{\prime}} t}^{\dagger} a_{\bar{k} t} a_{k t},
$$

can account for this [40, 41]]. The operators $a_{k t}^{\dagger}$ create a particle in the single-particle eigenstate $k$ of the mean-field Hamiltonian in the valence shell. The index $t$ indicates proton or neutron states and $\bar{k}$ denotes the time-reversed state of $k$. The operator $n_{k t}$ is the corresponding number-operator and $e_{k t}=e_{\bar{k} t}$ the single particle energy-eigenvalue. $G_{t}$ is the pairing strength for protons or neutrons. Proton-neutron pairing is not included, but this coupling contributes only in an important way for $N=Z$ nuclei [42]. As a consequence, the problem decouples for protons and neutrons. In the sequel only the neutron part of the model is considered, and the isospin index $t$ is dropped to ease the notations.

Based on algebraic techniques developed by Richardson [43], the pairing model can be solved exactly for an arbitrary set of single particle levels at zero temperature [44]. In practice it remains difficult to use these exact results to study the thermodynamics of the model, because the number of states needed in the ensemble increases very rapidly with increasing temperature. Thermodynamical properties have been studied using auxiliary-field QMC, which is free of sign problems for the pairing Hamiltonian of Eq. (34) when dealing with an even number of particles [24]. However, the present algorithm can consider nuclei with even and odd nucleon numbers. Note the auxiliary-field method scales as $\mathrm{O}\left(N_{s}^{3}\right)$ with $N_{s}$ the number considered single particle states, while a world-line algorithm scales linear with $N_{s}$.

When a nucleon occupies a single particle state $k$ and its time-reversed state $\bar{k}$ is unoccupied, the nucleon is said to be 'unaccompanied'. These states do not participate in the pair scattering by $H_{P}$. The mean-field plus pairing Hamiltonian can be rewritten as Eq. (1),

$$
\begin{aligned}
H & =H_{0}-V, \\
H_{0} & =\sum_{k} e_{k} n_{k}-\frac{G}{2} \sum_{k} b_{k}^{\dagger} b_{k}, \\
V & =\frac{G}{4} \sum_{k \neq k^{\prime}} b_{k}^{\dagger} b_{k^{\prime}} .
\end{aligned}
$$

The operators $b_{k}^{\dagger}=a_{k}^{\dagger} a_{k}^{\dagger}$ create a pair of nucleons in two timereversed states and satisfy hard-core boson commutation relations. In order to get the correct finite temperature properties, the possibility of changing the number of unaccompanied nucleons during the simulation should be incorporated. A path integral Monte Carlo method for the pairing Hamiltonian has been developed by Cerf and Martin [45, 46], but there the number of pairs remained fixed [24, 47]. This problem can now be overcome elegantly by adding an extra pair breaking term

$$
V_{\text {pert }}=\frac{G g}{2} \sum_{k} \sum_{k^{\prime} \neq k^{\prime \prime}}\left(b_{k}^{\dagger} a_{k^{\prime}} a_{k^{\prime \prime}}+H . c .\right),
$$

to the interaction part $V$ of Eq. 377. We define the worm operator as

$$
A=\frac{1}{\bar{N}} \sum_{k} n_{k}+\frac{1}{4} \sum_{k \neq k^{\prime}} b_{k}^{\dagger} b_{k^{\prime}}+\frac{g}{2} \sum_{k} \sum_{k^{\prime} \neq k^{\prime \prime}}\left(b_{k}^{\dagger} a_{k^{\prime}} a_{k^{\prime \prime}}+H . c .\right),
$$

with two extra parameters $\bar{N}$ and $g$ to be optimized. A term proportional to $V_{\text {pert }}$ is included in the worm operator, in order to satisfy condition Eq. 10]. This term will generate configurations with pair breaking interactions. However, it can occur 
that too many of these interactions are generated, though we are only interested in generating configurations with a different number of unaccompanied particles, but without interactions of the type Eq. (38). This can be prevented by imposing the constraint that a configuration can contain at most two pair breaking interactions of this type. Observables are only updated if there are no pair-breaking interactions in the configuration. This means that a number of Markov steps are needed in order to reach a new allowed configuration with a different number of unaccompanied particles. When $g$ of Eq. (39) is put equal to one, the percentage of diagonal configurations which contain no $V_{\text {pert }}$ interactions (see Eq. (38) is about $15 \%$. This is still efficient enough to sample the pairing Hamiltonian. There are a number of ways to increase the efficiency. First of all one can change the parameter $g$, hereby influencing the appearance of pair breaking interactions. One can also restrict the number of times the worm tries to insert a $V_{\text {pert }}$ interaction by allowing this only after a certain Markov time in which 'good' (i.e. without pair breaking interactions) configurations are sampled. One should also keep in mind that while a configuration contains pair breaking interactions, the worm itself is not necessarily of the pair breaking type. So a lot of Markov time is spend to change the configuration in a global way without removing the pair breaking interactions, leading to strong decorrelation.

The main physical properties of nuclei in the Iron region are modeled by a schematic mean-field plus pairing Hamiltonian. For the mean-field potential, we use a Woods-Saxon potential. Single particle energies are taken from Ref. [24]. A full $f p+s d g$ valence space is chosen. These single-particle states and energies are shown in Table III A pairing strength $G=16 / 56 \mathrm{MeV}$ is used. Due to the size of the model space a strength smaller than the suggested value of $20 \mathrm{MeV}$ per nucleon is used [24]. We have tested our code by comparing finite temperature results in a $f p$ valence space with the ones obtained via an exact diagonalization technique [48]. We show results of calculations with the valence shell given in Table III occupied by 10 and 11 valence neutrons. Figure 6 shows the expectation value of the neutron pairing-interaction operator $\left\langle H_{P}\right\rangle$ as a function of temperature. At low temperature, the pairing energies are much lower for the even number of neutrons. This can be understood by remarking that for an odd number of neutrons there is always at least one unpaired nucleon. At temperatures higher than $1 \mathrm{MeV}$, the pairing energies differ only slightly, because there is an increasing number of unpaired nucleons due to thermal excitation. This is also reflected in the specific heat (see Figure 77. A peak appears around $0.8 \mathrm{MeV}$ due to the development of pair correlations.

Because the worm operator conserves angular momentum, one can restrict the intermediate states to a specific value of the quantum numbers $J$ and $J_{z}$. This is not possible with the auxiliary-field QMC method. In our current implementation of the algorithm however, the occupation of each couple of time-reversed single-particle states $(k, \bar{k})$ is exactly known at all times. Because the unaccompanied particle number operator

$$
N^{u}=\sum_{k}\left(n_{k}-b_{k}^{\dagger} b_{k}\right)
$$

\begin{tabular}{|r|r|r|}
\hline \hline \multicolumn{3}{|c|}{ Single-particle energies (MeV) } \\
\hline Orbital & Protons & Neutrons \\
\hline $1 f_{7 / 2}$ & -4.1205 & -10.4576 \\
$2 p_{3 / 2}$ & -2.0360 & -8.4804 \\
$2 p_{1 / 2}$ & -1.2334 & -7.6512 \\
$1 f_{5 / 2}$ & -1.2159 & -7.7025 \\
$3 s_{1 / 2}$ & 4.7316 & -0.3861 \\
$2 d_{5 / 2}$ & 5.6562 & 0.2225 \\
$2 d_{3 / 2}$ & 6.1324 & 0.9907 \\
$1 g_{9 / 2}$ & 6.6572 & 0.5631 \\
\hline \hline
\end{tabular}

TABLE III: Single particle eigenstates of a Woods-Saxon potential, taken from Ref. [24]. The chosen valence space contains 42 states. The proton and neutron single particle energies (in $\mathrm{MeV}$ ) are shown on the right.

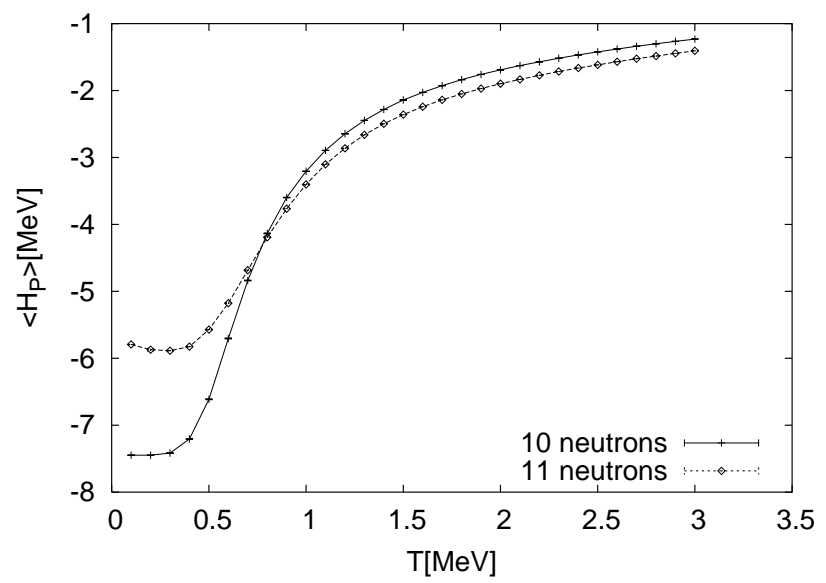

FIG. 6: Expectation value of the neutron part of the pairinginteraction operator as a function of temperature. The pairing strength $G_{n}$ is equal to $16 / 56 \mathrm{MeV}$. We consider 10 and 11 neutrons in the $f p+s d g$ valence space (see Tabel III). At temperatures below $0.5 \mathrm{MeV}$ the pairing energy is much lower for the even neutron number.

commutes with the angular momentum projection operator $J_{z}$ (but not with $J^{2}$ ), our current code allows restricting the simulation to configurations with a fixed $J_{z}$. Work on extending this technique to full J-projection is in progress.

When the projection on $J_{z}$ was turned on, we included an extra global step in order to get a good convergence at the lowest temperatures. This extra global change allows for one or two unaccompanied nucleons (which block the state they occupy) to move to other states, and can occur whenever the worm is diagonal. First an unaccompanied nucleon at a blocked state $l$ is chosen at random. A 'non-blocked' pair of states $(k, \bar{k})$ is then chosen with probability

$$
P(k)=e^{\int_{0}^{\beta}\left(n_{k}(t)+n_{\bar{k}}(t)-1\right) e_{k} d t} / N_{l},
$$

with $n_{k}(t)$ the occupation number of state $k$ at imaginary time $t$, and $N_{l}$ a normalization factor. The subscript $l$ indicates that the norm is determined for a configuration containing a blocked state $l$. The idea behind Eq. 41) is to get a probability distribution $P(k)$ which is peaked around the Fermi level, 


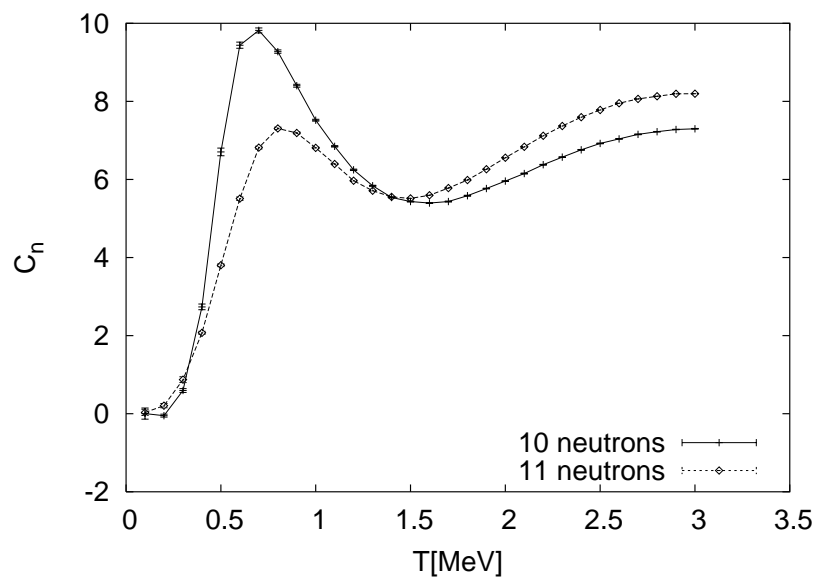

FIG. 7: The neutron specific heat $C_{n}$ as a function of temperature for 10 and 11 neutrons in the full $f p+s d g$ valence space (see Table III). Calculations were performed at a constant neutron pairing strength $G_{n}=16 / 56 \mathrm{MeV}$.

but other distributions can be chosen as well. The interchange of the occupations of the blocked pair of states $(l, \bar{l})$ and the non-blocked pair $(k, \bar{k})$ over the whole imaginary time interval $\beta$, is accepted with probability

$$
p=\min \left(1, \frac{N_{l}}{N_{k}}\right) .
$$

The acceptance factor for the case when the occupations of two pairs of non-blocked and blocked states are interchanged, can be constructed in a similar way. The extra step has a high acceptance rate, but is only necessary to enhance decorrelation at very low temperature when a $J_{z}$-projection is included. At higher temperatures the unaccompanied nucleons move efficiently from state to state via the last worm piece in Eq. (39). Figure 8 shows total energies after $J_{z}$-projection at low temperature. Calculations were performed for ten neutrons moving in the model space listed in Table III The neutron pairing strength is again $G_{n}=16 / 56 \mathrm{MeV}$. The figure also shows exact energy eigenvalues for $J_{z}=0$ to $J_{z}=7$. These were calculated via a technique explained in Ref. [44]. The lowest $J_{z}=1,2$ and the lowest $J_{z}=3,4$ states are degenerate. For low enough values of $T$ the finite temperature results clearly converge to the ground states within the considered ensembles.

Note that we can compare with exact solutions because the pairing strength was taken constant for all levels. Our QMC method allows to solve pairing models with a single particle state dependent pairing strength $G_{k k^{\prime}}$, for which no algebraic solutions are available. Taking in mind the method is applicable for even and odd nucleon systems and allows angular momentum symmetry projections, this could greatly extend the applicability of the pairing model.

\section{CONCLUSIONS AND OUTLOOK}

We have set up a quantum Monte Carlo method with a nonlocal loop updating scheme starting from a local worm op-

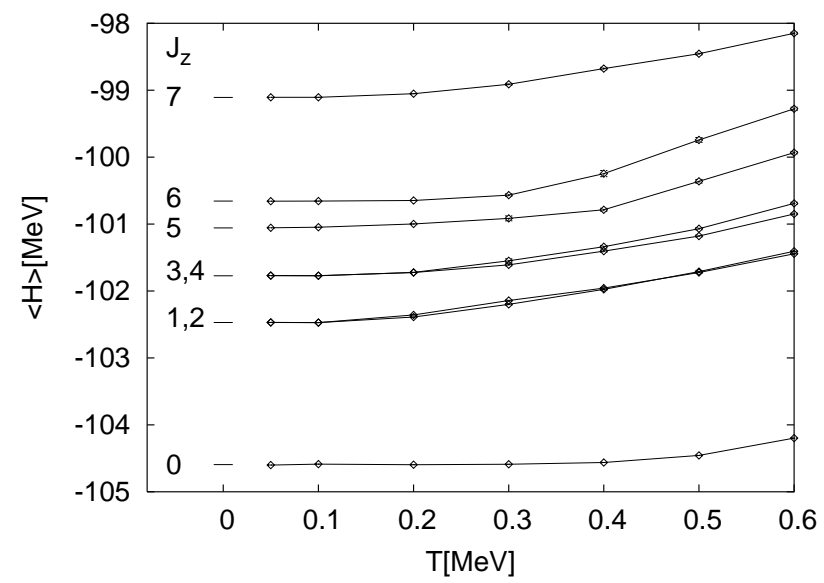

FIG. 8: $J_{z}$ projected internal energies as a function of temperature. The values of $J_{z}$ from 0 to 7 are indicated on the left. A clear convergence to exact zero temperature results calculated via techniques explained in Ref. [44] can be seen.

erator in the path integral approach. This method allows to sample configurations with specific symmetries and, in particular, to sample the canonical ensemble. It leads to a very efficient sampling scheme with all moves accepted and without 'bounces' or critical slowing down near second order phase transitions. We have proven detailed balance and tested ergodicity. Our method opens new perspectives for the study of quantum many-body systems where particle number and other symmetries play an important role. It can be applied to bosons, to fermions in absence of a sign problem and to non-frustrated spin systems at fixed magnetization. We have demonstrated this by simulating the Bose-Hubbard model and a nuclear pairing model. The equal-time one-body Green's function can be evaluated with high efficiency. When nonequal time observables are required, the current method can in principle still be combined with conventional non-local worm steps. There is still a lot of freedom in choosing the algorithm parameters, which can be used to optimize the algorithm. For the Bose-Hubbard model we compared the efficiency of our algorithm (with different parameter sets) with a directed loop SSE code. Though one should always be careful when comparing different algorithms, we have strong indications that our method is very efficient. We have simulated a pairing model for even and odd particle numbers. Our finite temperature results clearly supplement algebraic methods and other QMC methods. Furthermore, a projection on angular momentum symmetries can be included. We have demonstrated this by showing $J_{z}$-projected results. A work on full $J$-projection is in progress.

The authors wish to thank K.Heyde, J. Dukelsky, S. Wessel, M. Troyer and S. Trebst for interesting discussions and the Fund for Scientific Research - Flanders (Belgium), the Research Board of the University of Ghent and N.A.T.O. for financial support. 
[1] N. Metropolis, A.R. Rosenbluth, M.N. Rosenbluth, A.H. Teller and E. Teller, J. of Chem. Phys. 21, 1087 (1953).

[2] W. von der Linden, Phys. Rep. 220, 53 (1992).

[3] U. Wolff, Phys. Rev. Lett. 62, 361 (1989).

[4] R.H. Swendsen and J.-S. Wang, Phys. Rev. Lett. 58, 86 (1987).

[5] H.G. Evertz, G. Lana and M. Marcu, Phys. Rev. Lett. 70, 875 (1993).

[6] H.G. Evertz, Adv. Phys. 52, 1 (2003).

[7] N. Kawashima and K. Harada, J. Phys. Soc. Jpn. 73, 1379 (2004).

[8] M. Suzuki, Prog. of Theor. Phys. 56, 1454 (1976).

[9] H.F. Trotter, Proc. Am. Math. Soc. 10, 545 (1959).

[10] B.B. Beard and U.-J. Wiese, Phys. Rev. Lett. 77, 5130 (1996).

[11] N.V. Prokof'ev, B.V. Svistunov and I.S. Tupitsyn, Phys. Lett. A, 238, 253 (1998);Pis'ma v Zh. Eks. Teor. Fiz. 64, 853 (1996).

[12] N.V. Prokof'ev, B.V. Svistunov and I.S. Tupitsyn, Sov. Phys. JETP 87, 310 (1998).

[13] A.W. Sandvik and J. Kurkijärvi, Phys. Rev. B 43, 5950 (1991).

[14] A.W. Sandvik, Phys. Rev. B 59, R14157 (1999).

[15] A. Dorneich and M. Troyer, Phys. Rev. E 64, 066701 (2001).

[16] O.F. Syljuåsen and A.W. Sandvik, Phys. Rev. E 66, 046701 (2002).

[17] O.F. Syljuåsen, Phys. Rev. E 67, 046701 (2003).

[18] L. Pollet, S.M.A. Rombouts, K. Van Houcke and K. Heyde, Phys. Rev. E 70, 056705 (2004).

[19] P. Roos and S. Miyashita, Phys. Rev B 59, 13782 (1999).

[20] S. Sachdev, Quantum Phase Transitions, Cambridge University Press, Cambridge (2000).

[21] J. Dukelsky and G. Sierra, Phys. Rev. Lett. 83, 172 (1999).

[22] J. von Delft and D.C. Ralph, Phys. Rep. 345, 61 (2001).

[23] S.E. Koonin, D.J. Dean and K. Langanke, Phys. Rep. 278, 1 (1997).

[24] S. Rombouts, K. Heyde and N. Jachowicz, Phys. Rev. C 58, 3295 (1998).

[25] S.M.A. Rombouts, K. Van Houcke and L. Pollet, unpublished.

[26] J.E. Hirsch, D.J. Scalapino, R.L. Sugar and R. Blankenbecler,
Phys. Rev. Lett. 47, 1628 (1981).

[27] S. Rombouts and K. Heyde, Phys. Stat. Sol. B 237, 99 (2003).

[28] W.K. Hastings, Biometrika 57, 97 (1970).

[29] D. Jaksch, C. Bruder, J.I. Cirac, C.W. Gardiner, and P. Zoller, Phys. Rev. Lett. 81, 3108 (1998).

[30] M. Greiner, O. Mandel, T. Esslinger, T.W. Hänsch and I. Bloch, Nature 415, 39 (2002).

[31] M.P.A. Fisher, P.B. Weichman, G. Grinstein and D.S. Fisher, Phys. Rev. B 40, 546 (1989).

[32] V.L. Berezinskii, Sov. Phys. - JETP 32, 493 (1970).

[33] J.M. Kosterlitz and D.J. Thouless, J. Phys. C 6, 1181 (1973).

[34] F. Wang and D.P. Landau, Phys. Rev. Lett. 86, 2050 (2001); Phys. Rev. E 64, 056101 (2001).

[35] E.L. Pollock and D.M. Ceperley, Phys. Rev. B 36, 8343 (1987).

[36] L. Pollet, PhD thesis, Ghent University, unpublished.

[37] A.W. Sandvik, R.R.P. Singh and D.K. Campbell, Phys. Rev. B 56, 14510 (1997).

[38] B.S. Pudliner, V.R. Pandharipande, J. Carlson and R.B. Wiringa, Phys. Rev. Lett. 74, 4396 (1995).

[39] W.E. Ormand, Phys. Rev. C 56, R1678 (1997); H. Nakada and Y. Alhassid, Phys. Rev. Lett. 79, 2939 (1997).

[40] P. Ring and P. schuck, The Nuclear Many-Body Problem (Springer, New York, 1980), p. 217.

[41] D.J. Dean and M. Hjorth-Jensen, Rev. Mod. Phys. 75, 607 (2003).

[42] K. Langanke, D.J. Dean, S.E. Koonin and P.B. Radha, Nucl. Phys. A 613, 253 (1997).

[43] R. Richardson and N. Sherman, Nucl. Phys. 52, 221 (1964); R.W. Richardson, Phys. Rev. 141, 949 (1966).

[44] S. Rombouts, D. Van Neck and J. Dukelsky, Phys.Rev. C 69, 061303(R) (2004).

[45] N. Cerf and O. Martin, Phys. Rev. C 47, 2610 (1993).

[46] N.J. Cerf, Phys. Rev. Lett. 76, 2420 (1996).

[47] S. Rombouts and K. Heyde, Phys. Rev. Lett. 80, 885 (1998).

[48] R. Rossignoli, Phys. Rev. C 54, 1230 (1996). 